\title{
Uniportal video-assisted thoracoscopic surgery following neoadjuvant chemotherapy for locally-advanced lung cancer
}

Zhiqiang Yang and Chunbo Zhai ${ }^{*}$ (D)

\begin{abstract}
Background: Several retrospective studies have confirmed that video-assisted thoracoscopic surgery (VATS) following neoadjuvant chemotherapy is a safe and feasible treatment for advanced non-small cell lung cancer patients. As a minimally invasive technique, VATS usually leads to better clinical outcomes and better compliance with adjuvant treatment than conventional thoracotomy. Uniportal VATS (U-VATS) as an alternative option to conventional multi-port VATS has attracted much attention recently because reduced number and size of incisions may help to decrease inflammatory response and reduce postoperative pain for patients. However, rarely studies have reported the application of U-VATS following neoadjuvant chemotherapy for the treatment of advanced lung cancer patients.
\end{abstract}

Methods: A total of 29 lung cancer patients undergoing VATS following neoadjuvant chemotherapy were included in this study. The clinical data of these patients were retrospectively analyzed, including the preoperative neoadjuvant chemotherapy plan, surgical effect, postoperative complications, operation time, operative blood loss, number of lymph nodes dissected and postoperative mortality.

Results: All patients underwent VATS following two cycles of neoadjuvant chemotherapy. Among these patients, 26 completed U-VATS, two were converted to triple-port VATS, and one was converted to open thoracotomy. The operation time ranged from 120 min to 300 min (mean: $160 \pm 38.5$ min); the operative blood loss was 50-500 ml (mean:167.8 \pm 78 . $4 \mathrm{ml}$ ); the number of lymph nodes dissected was 16-28 (mean: 21.9 \pm 3.7); the postoperative drainage time was 3-13 d (mean: $5.6 \pm 1.9 \mathrm{~d}$ ); and the postoperative hospital stay was 6-16 d (7.7 $\pm 1.9 \mathrm{~d}$ ). Postoperative complications occurred in five (17.2\%) patients, including three cases of respiratory infection, one case of air leakage (more than two weeks), and one case of wound infection. In addition, the 30- and 90-day postoperative mortality was zero.

Conclusion: U-VATS following neoadjuvant chemotherapy is feasible and safe for the treatment of advanced lung cancer patients.

Keywords: Lung cancer, Neoadjuvant chemotherapy, Uniportal video-assisted thoracoscopic surgery (U-VATS)

\footnotetext{
* Correspondence: zhaicbmd@gmail.com

Department of Thoracic Surgery, Weifang People's Hospital, Shandong

Province, Weifang 261041, China
}

(c) The Author(s). 2018 Open Access This article is distributed under the terms of the Creative Commons Attribution 4.0 International License (http://creativecommons.org/licenses/by/4.0/), which permits unrestricted use, distribution, and reproduction in any medium, provided you give appropriate credit to the original author(s) and the source, provide a link to the Creative Commons license, and indicate if changes were made. The Creative Commons Public Domain Dedication waiver (http://creativecommons.org/publicdomain/zero/1.0/) applies to the data made available in this article, unless otherwise stated. 


\section{Background}

Recent reports indicate that preoperative neoadjuvant chemotherapy or chemoradiotherapy can improve the completeness of resection and survival in patients with limited small-cell lung cancer (SCLC) and advanced nonsmall cell lung cancer (NSCLC) [1, 2]. However, tissue adhesions and increased vascular fragility after neoadjuvant chemotherapy could increase the risk of surgery, the incidence of postoperative complications and mortality [3].

Conventional open thoracotomy is associated with severe trauma and a lengthy recovery. Its tolerability in patients is very poor after neoadjuvant chemotherapy [4]. However, VATS lobectomy has the advantages of minimal trauma, less pain, quicker recovery and fewer complications [5]. Several retrospective studies have confirmed that VATS following neoadjuvant chemotherapy is a safe and feasible treatment for advanced NSCLC patients [6, 7]. Furthermore, uniportal VATS as an alternative option to conventional multi-port VATS has attracted much attention recently [8]. However, rarely studies have reported the application of U-VATS following neoadjuvant chemotherapy for the treatment of locally- advanced lung cancer patients [9]. The aim of the current study is to investigate the safety and feasibility of U-VATS following neoadjuvant chemotherapy for the treatment of advanced lung cancer patients.

\section{Methods}

\section{Patient information}

From June 2015 to October 2016, a total of 29 lung cancer patients (18 male and 11 female) with stage IIB-IIIB were included in this study. The mean age of these patients was $53.8 \pm 6.4$ years (range from 47 to 66 years). All patients were recruited from Weifang People's Hospital in China. The detailed clinical and pathological features of these patients are shown in Table 1.

All patients were diagnosed before preoperative chemotherapy. The histopathological diagnosis was confirmed by fibrobronchoscopy and percutaneous needle biopsy. The NSCLC types included adenocarcinoma ( $n=16,55.2 \%)$, squamous cell carcinoma $(n=8,27.6 \%)$ and SCLC $(n=5$, $17.2 \%)$. The clinical stages were assessed and confirmed through preoperative positron emission tomographycomputed tomography (PET-CT). The preoperative examination also included bronchoscopy, brain MRI and cardiopulmonary function tests. Tumor, node and metastasis (TNM) staging was performed based on the 2009 UICC staging criteria (7th edition) [10], with eight cases of stage IIB (27.6\%), 18 cases of stage IIIA (62.1\%) and three cases of stage IIIB (10.3\%).

\section{Neoadjuvant chemotherapy}

The neoadjuvant chemotherapy treatment regimens of patients were selected based on the pathologic type. A
Table 1 Patient's clinical and pathological features

\begin{tabular}{ll}
\hline Characteristic & No. (\%) \\
\hline Gender & $18(62.1)$ \\
Male & $11(37.9)$ \\
Female & \\
Histological types & $16(55.2)$ \\
Adenocarcinoma & $8(27.6)$ \\
Squamous cell carcinoma & $5(17.2)$ \\
Small cell carcinoma & \\
Clinical TNM staging & $8(27.6)$ \\
IIB & $18(62.1)$ \\
IIIA & $3(10.3)$ \\
IIIB & \\
Clinical TNM staging approach & $29(100)$ \\
PET-CT & $19(65.5)$ \\
Definite diagnosis of histological types & $10(34.5)$ \\
CT-guided biopsy & \\
Bronchoscopy & \\
\hline
\end{tabular}

total of sixteen adenocarcinoma patients received DP (docetaxel + cisplatin), eight squamous cell carcinoma patients received GP (gemcitabine + cisplatin), and five SCLC patients received EC (Etoposide + carboplatin, Table 2). All patients underwent two cycles of preoperative neoadjuvant chemotherapy. Four weeks after neoadjuvant chemotherapy treatment, the patients underwent another chest enhanced CT. If no lesion progression or reduction in staging was confirmed by CT, VATS was performed. The average time from the end of the neoadjuvant chemotherapy therapy to the operation was 35.5 $\pm 2.8 \mathrm{~d}$.

\section{VATS technique}

The patients received general anesthesia in a contralateral supine position and underwent double-lumen endotracheal intubation. The surgeon and an assistant stood on the ventral side of the patient, and another assistant

Table 2 Neoadjuvant chemotherapy treatment regimens and evaluation of their effectiveness

\begin{tabular}{|c|c|c|c|}
\hline \multirow{2}{*}{$\begin{array}{l}\text { Neoadjuvant } \\
\text { treatment } \\
\text { regimen }\end{array}$} & \multicolumn{3}{|c|}{ Effectiveness, n (\%) } \\
\hline & $C R$ & PR & SD \\
\hline GP & - & 12 & 4 \\
\hline DP & - & 5 & 3 \\
\hline EC & - & 5 & - \\
\hline \multicolumn{4}{|c|}{$\begin{array}{l}\text { GP (gemcitabine + cisplatin): gemcitabine } 1000 \mathrm{mg} / \mathrm{m}^{2} \text {, intravenous infusion, } \\
\mathrm{d} 1 \text {, d8; total cisplatin } 90 \mathrm{mg} / \mathrm{m}^{2} \text {, intravenous infusion, d2-5, } 21 \text { days as one } \\
\text { cycle; DP (docetaxel + cisplatin), docetaxel injection, intravenous infusion, } \\
75 \mathrm{mg} / \mathrm{m}^{2}, \mathrm{~d} 1 \text {, total cisplatin } 90 \mathrm{mg} / \mathrm{m}^{2} \text {, intravenous infusion, } \mathrm{d} 2-5,21 \text { days as } \\
\text { one cycle; EC (Etoposide,VP- } 16+\text { carboplatin), carboplatin intravenous infusion, } \\
\text { d1, AUC (area under the curve) method to calculate the carboplatin dose, } \\
\text { AUC }=5.5 \text {, total etoposide } 100 \mathrm{mg} / \mathrm{m}^{2}, \mathrm{~d} 2-6,21 \text { days as one cycle }\end{array}$} \\
\hline
\end{tabular}


stood on the dorsal side of the patient. The operation hole was typically positioned between the middle axillary line and the anterior axillary line. In addition, an upper or lower lobectomy was usually performed via the 5th intercostal space, and a middle lobectomy was usually performed at the 4 th intercostal space, with a $3-5-\mathrm{cm}$ long incision. To enlarge the incision, a lap-protector was used rather than a rib spreader. The lung fissure and blood vessels were carefully separated referenced to their development on the upper lobe. In patients with an intractable left upper pulmonary artery, the pulmonary trunk was preferentially separated. In addition, the middle and lower lobectomies were performed in the following order: veins, lobar bronchia, arteries and lung fissure. Simultaneously, the lymph nodes in stations 10 and 11 were cleared away. After the lobectomy, a mediastinal lymph node dissection was systematically performed (stations 4, 5, 6, 7, 8, and 9 on the left; stations $2,3,4,7,8$, and 9 on the right). Additionally, a $28 \mathrm{~F}$ drainage tube was placed through the surgical incision after the operation.

\section{Statistical analysis}

Statistical analyses were applied via SPSS software (version 17.0). Student's t-test was performed to compare measurement data, whereas the $\chi^{2}$ test was utilized to compare enumeration data. A $P$-value of less than 0.05 was considered statistically significant (two sides).

\section{Results}

\section{Clinical efficacy and toxic effects of neoadjuvant chemotherapy therapy}

All 29 patients completed two cycles of preoperative neoadjuvant chemotherapy. Among the 29 patients who received neoadjuvant chemotherapy, 12 adenocarcinoma, 5 squamous cell carcinoma and 5 SCLC patients were radiologic partly response (PR), and another four adenocarcinoma and three squamous cell carcinoma patients exhibited a stable disease (SD) after neoadjuvant chemotherapy therapy (Table 2). Among the five SCLC patients, two exhibited a pathological complete response (CR, Table 2).

\section{Surgical results and complications}

Among the 29 patients, 28 underwent successful VATS with systematic lymph node dissection. Among these patients, 26 underwent U-VATS, two were converted to triple-port VATS because of compact perivascular lymph nodes, and one was converted to open thoracotomy due to bleeding during the operation. The operation time ranged from $120 \mathrm{~min}$ to $300 \mathrm{~min}$ (mean: $160 \pm 38$. $5 \mathrm{~min}$ ); the operative blood loss was $50-500 \mathrm{ml}$ (mean: $167.8 \pm 78.4 \mathrm{ml}$ ); the number of the dissected lymph nodes was $16-28$ (mean: $21.9 \pm 3.7$ ); the postoperative drainage time was 3-13 d (mean: $5.6 \pm 1.9 \mathrm{~d}$ ); and the postoperative hospital stay was 6-16 d $(7.7 \pm 1.9 \mathrm{~d}$, Table 3). Postoperative complications occurred in five (17.2\%) patients, including three cases of respiratory infection, one case of air leakage (more than two weeks), and one case of wound infection. In addition, both the 30- and 90-day postoperative mortality were zero.

\section{Discussion}

In recent years, VATS lobectomy has been used worldwide and has been increasingly used to treat locally- advanced NSCLC $[6,11]$. However, VATS following neoadjuvant chemotherapy is still a challenge. As reported, the incidence of postoperative complications after neoadjuvant chemotherapy or chemoradiotherapy is as high as 35-43. $5 \%[3,12]$. In addition, the main cause of postoperative complications was tissue adhesions, indistinct interface and increased fragility of the blood vessels after neoadjuvant chemotherapy. Hence, safety after neoadjuvant chemotherapy is always the focus of surgeons. With the development of the thoracoscopic technique, the safety and feasibility of complete VATS (cVATS) following neoadjuvant chemotherapy have been increasingly reported $[2,6]$. Huang et al [6] reported that the incidence of postoperative complications and perioperative mortality after cVATS was $9.5 \%$ and $2.4 \%$, respectively, which was consistent with the outcomes of open thoracotomy.

\begin{tabular}{ll}
\multicolumn{2}{l}{ Table 3 Surgical outcomes of patients undergoing U-VATS } \\
\hline Item & No. (\%) or range \\
\hline Lobe resection & $9(31.1)$ \\
Right upper lobectomy & $2(6.9)$ \\
Right middle lobectomy & $7(24.1)$ \\
Right lower lobectomy & $5(17.2)$ \\
Left upper lobectomy & $6(20.7)$ \\
Left lower lobectomy & \\
Postoperative TNM staging & $2(6.9)$ \\
No tumor & $1(3.5)$ \\
IA & $3(10.3)$ \\
IB & $7(24.1)$ \\
IIA & $10(34.5)$ \\
IIB & $5(17.2)$ \\
IIIA & $1(3.5)$ \\
IIIB & $160 \pm 38.5$ \\
Operation time (min) & $167.8 \pm 78.4$ \\
Intraoperative blood loss (ml) & $21.9 \pm 3.7$ \\
No. of lymph nodes dissected & $5.6 \pm 1.9$ \\
Postoperative chest tube duration (days) & $7.7 \pm 1.9$ \\
Postoperative hospital stay (days) & $35.5 \pm 2.8$ \\
\hline Time to chemotherapy (days) &
\end{tabular}


U-VATS is a newly developed technology based on VATS. Since Rocco et al [13] first reported the use of lung wedge resection via U-VATS, the indications of U-VATS in diagnosis and treatment of lung disease have been extended. Very recently, Gonzalez-Rivas et al [14-16] used U-VATS to complete various complex and highly difficult procedures, including segmentectomy, bronchial sleeve lobectomy and double-sleeve (vascular and bronchial) lobectomy. Furthermore, Gonzalez-Rivas et al [9] and Fan et al [17] confirmed the safety and feasibility of U-VATS for advanced NSCLC patients. However, the use of U-VATS in treating advanced NSCLC following neoadjuvant chemotherapy, is still a challenge for most thoracic surgeons.

The mean operation time was $160 \pm 38.5 \mathrm{~min}$, the operative blood loss was $167.8 \pm 78.4 \mathrm{ml}$ and the number of lymph nodes dissected was $21.9 \pm 3.7$, which are all consistent with those reported by previously published studies $[9,17,18]$. However, the mean postoperative drainage time $(5.6 \pm 1.9 \mathrm{~d})$ and postoperative hospital stay $(7.7 \pm 1.9$ d) were longer than those reported by previously published studies $[9,17,18]$. The cause of the longer times may be related to the standard for removing the chest tube. In our current study, the chest tube was removed when the drainage was less than $100 \mathrm{ml} / 24 \mathrm{~h}$. However, Zhang et al [19] compared the safety and feasibility of early chest tube removal (removal of chest tube when drainage is less than $300 \mathrm{ml} / 24 \mathrm{~h}$ ) to the traditional management method (The chest tube was removed when the drainage is less than $100 \mathrm{ml} / 24 \mathrm{~h}$ ), and they confirmed that early removal of the chest tube after lobectomy is feasible and safe. Additionally, the early removal of the chest tube could lead to a shorter hospital stay and reduce morbidity without increasing the risk of complications. Hence, we hypothesized that the traditional standard for chest tube removal may have contributed to the longer postoperative drainage time and hospital stay.

In our current study, the incidence of and mortality from postoperative complications were $17.2 \%$ and zero, respectively. Lung infection was typically the most common postoperative complication. Villamizar et al [20] reported that prior chemotherapy could predict increased complications based on a multivariable analysis, whereas Gonzalez-Rivas et al [9] and Fan et al [17] showed that VATS in advanced NSCLC patients after neoadjuvant chemotherapy was not associated with an increase in the incidence of postoperative complications. In addition, our results were similar as those reported by GonzalezRivas et al [9] and Fan et al. [17] They also reported that reduced access trauma, a better view and the skills and experience of the surgeon may contribute to the low incidence of postoperative complications.

Among the 29 patients who underwent VATS following neoadjuvant chemotherapy, two $(6.9 \%)$ patients were converted to triple-port VATS, and one (3.4\%) patient was converted to open thoracotomy, which were consistent with previously studies $[6,9,17]$. Tissue adhesions, indistinct interface and increased fragility of the blood vessels after neoadjuvant chemotherapy were significant causes of operative blood loss. Additionally, mistakes during the operation could lead to blood loss as well. For example, injury of the vascular wall from violent dissection of the blood vessels or a forced pass of the stapler may cause avulsion of the vascular roots when the length of the dissected blood vessels was insufficient. When rupture of the veins and bronchus occurs, violent tension from the assistants could also cause the rupture of blood vessels.

Another cause of conversion to thoracotomy was perivascular and peribronchial lymph node calcification. As Park et al [21] reported in a retrospectively study, $41 \%$ of conversions may be due to the anthracofibrosis of hilar lymph nodes or hilar adhesions. They also analyzed the CT scans of patients and found that $71 \%$ of patients suffered from different levels of hilar lymph node calcification. In our current study, two patients were not converted to open thoracotomy for the compact perivascular lymph nodes, but the operation could not be completed by U-VATS; two additional ports were added to finish the lobectomy. In addition, Wang et al [22] gained proximal and distal control of the artery before cutting the arteries and the application of a stapler at the end was an important way to ensure the safety and reliability of U-VATS in treating calcified lymph nodes.

To reduce the occurrence of operative blood loss, the following strategies were utilized: (I) Treat the part with the lowest risk of bleeding first during surgery, and avoid adherence to a certain order; (II) Dissect blood vessels extensively and expand the vascular space. Additionally, a silk thread could be used to hold the vessels when stapler was difficult to use; (III) When the risk of bleeding is high, freeing the root of the pulmonary artery in advance and temporarily blocking the artery could not only reduce the occurrence of operative blood loss but also reduce the time required to repair the ruptured blood vessels; (IV) For small blood vessels, cut after silk thread ligation was safer and more convenient; and (V) When there is gap fuzziness and dense adhesion between the blood vessels and bronchia such that blunt separation is difficult, we can temporarily cut the bronchia and suture the bronchial stump or use a stapler at the end after lung lobectomy.

Some limitations were unavoidable in this study. First, this was a retrospective study and thus it lacked prospective randomized controls. Second, the clinical stages of patients were assessed and confirmed through preoperative PET-CT without verification by endobronchial ultrasound or mediastinoscopy, which might generate 
biases on the accuracy of the clinical stages. As reported, PET-CT has high sensitivity, specificity and diagnostic accuracy in the mediastinal staging of patients, but PET$\mathrm{CT}$ alone cannot judge the extent of mediastinal lymph node involvement [23]. Hence, the combination of PET$\mathrm{CT}$ and other examination methods could improve the diagnostic accuracy of clinical stages. Third, due to the limited number of patient samples in this study, our conclusions in the manuscript need to be further validated with more patient samples in the future.

\section{Conclusions}

In conclusion, our current study further confirmed the safety and feasibility of U-VATS following neoadjuvant chemotherapy for the treatment of advanced lung cancer patients considering the incidence of and mortality from operative and postoperative complications. Extensive experience, a solid foundation for VATS and rational operation procedures guarantee the successful completion of U-VATS. However, whether U-VATS could be more widely used and whether U-VATS is more advantageous than multiportal VATS or open thoracotomy still require further study.

\section{Abbreviations}

CR: complete response; cVATS: complete VATS; DP: docetaxel + cisplatin; EC: Etoposide + carboplatin; GP: gemcitabine + cisplatin; NSCLC: non-small cell lung cancer; PET-CT: positron emission tomography-computed tomography; PR: partly response; SCLC: small-cell lung cancer; SD: stable disease; TNM: Tumor, node and metastasis; U-VATS: Uniportal VATS; VATS: video-assisted thoracoscopic surgery

\section{Funding}

This study was supported and funded by the departmental budget.

\section{Availability of data and materials}

All data generated or analyzed during the current study are included in this published article.

\section{Authors' contributions}

CBZ designed the study. ZQY and CBZ collected and analyzed the data, interpreted the results and wrote the paper. Both authors have read and approved the final version of the manuscript.

\section{Ethics approval and consent to participate}

The study protocol was approved by the Ethics Committee of Weifang People's Hospital, and both the clinicians and patients provided signed informed consent.

\section{Consent for publication}

Patients provided consent for the publication of data about their health and health-related outcomes.

\section{Competing interests}

The authors declare that they have no competing interests.

\section{Publisher's note}

Springer Nature remains neutral with regard to jurisdictional claims in published maps and institutional affiliations.
Received: 16 January 2018 Accepted: 4 April 2018

Published online: 24 April 2018

\section{References}

1. Scagliotti GV, Pastorino U, Vansteenkiste JF, et al. Randomized phase III study of surgery alone or surgery plus preoperative cisplatin and gemcitabine in stages IB to IIIA non-small-cell lung cancer. J Clin Oncol. 2012;30:172-8.

2. $\mathrm{Xu} \mathrm{YJ}$, Zheng $\mathrm{H}$, Gao W, et al. Is neoadjuvant chemotherapy mandatory for limiteddisease small-cell lung cancer? Interact Cardiovasc Thorac Surg. 2014;19:887-93.

3. Venuta F, Anile M, Diso D, et al. Operative complications and early mortality after induction therapy for lung cancer. Eur J Cardiothorac Surg. 2007;31:714-7.

4. Flores RM, Park BJ, Dycoco J, et al. Lobectomy by video-assisted thoracic surgery (VATS) versus thoracotomy for lung cancer. J Thorac Cardiovasc Surg. 2009:138:11-8.

5. Cao C, Manganas C, Ang SC, Yan TDA. Meta-analysis of unmatched and matched patients comparing video-assisted thoracoscopic lobectomy and conventional open lobectomy. Ann Cardiothorac Surg. 2012;1:16-23.

6. Huang J, Xu X, Chen H, et al. Feasibility of complete video-assisted thoracoscopic surgery following neoadjuvant therapy for locally advanced non-small cell lung cancer. J Thorac Dis. 2013;5:S267-73.

7. Yang CF, Meyerhoff RR, Mayne NR, et al. Long-term survival following open versus thoracoscopic lobectomy after preoperative chemotherapy for nonsmall cell lung cancer. Eur J Cardiothorac Surg. 2016;49:1615-23.

8. Sihoe AD. The evolution of minimally invasive thoracic surgery: implications for the practice of uniportal thoracoscopic surgery. J Thorac Dis. 2014;6:S604-17.

9. Gonzalez-Rivas D, Fieira E, Delgado M, Mendez L, Fernandez R, de la Torre MI. Uniportal thoracoscopic surgery a feasible approach for advanced stages of non-small cell lung cancer? J Thorac Dis. 2014;6:641-8.

10. Giroux DJ, Rami-Porta R, Chansky K, et al. The IASLC lung cancer staging project: data elements for the prospective project. J Thorac Oncol. 2009;4:679-83.

11. Park BJ, Yang HX, Woo KM, Sima CS. Minimally invasive (robotic assisted thoracic surgery and video-assisted thoracic surgery) lobectomy for the treatment of locally advanced non-small cell lung cancer. J Thorac Dis. 2016;8(Suppl 4):S406-13.

12. Fujita S, Katakami N, Takahashi Y, et al. Postoperative complications after induction chemoradiotherapy in patients with non-small-cell lung cancer. Eur J Cardiothorac Surg. 2006;29:896-901.

13. Rocco G, Martin-Ucar A, Passera E. Uniportal VATS wedge pulmonary resections. Ann Thorac Surg. 2004;77:726-8.

14. Gonzalez-Rivas D, Fieira E, Mendez L, Garcia J. Single-port video-assisted thoracoscopic anatomic segmentectomy and right upper lobectomy. Eur J Cardiothorac Surg. 2012;42:e169-71.

15. Gonzalez-Rivas D, Fernandez R, Fieira E, Rellan L. Uniportal video-assisted thoracoscopic bronchial sleeve lobectomy: first report. J Thorac Cardiovasc Surg. 2013;145:1676-7.

16. Gonzalez-Rivas D, Delgado M, Fieira E, Fernandez R. Double sleeve uniportal video-assisted thoracoscopic lobectomy for non-small cell lung cancer. Ann Cardiothorac Surg. 2014;3:E2.

17. Fan J, Yao J, Wang Q, Chang Z. Safety and feasibility of uniportal videoassisted thoracoscopic surgery for locally advanced non-small cell lung cancer. J Thorac Dis. 2016;8:3543-50.

18. Mu JW, Gao SG, Xue Q, et al. A matched comparison study of uniportal versus triportal thoracoscopic lobectomy and sublobectomy for early-stage nonsmall cell lung cancer. Chin Med J. 2015;128:2731-5.

19. Zhang Y, Li H, Hu B, Hou SC, Li T, Miao JB, et al. Early removal of the chest tube after lobectomies: a prospective randomized control study. Chin J Surg. 2013;51:533-7.

20. Villamizar NR, Darrabie M, Hanna J, et al. Impact of T status and N status on perioperative outcomes after thoracoscopic lobectomy for lung cancer. J Thorac Cardiovasc Surg. 2013:145:514-21.

21. Park JS, Kim HK, Choi YS, Kim J, Shim YM, Kim K. Unplanned conversion to thoracotomy during video-assisted thoracic surgery lobectomy does not compromise the surgical outcome. World J Surg. 2011;35:590-5.

22. Wang GS, Wang J, Rao ZP, Ding GG, Wang Z. Uniportal complete videoassisted thoracoscopic surgery lobectomy with partial pulmonary arterioplasty for lung cancer with calcified lymph node. J Thorac Dis. 2015;7:2366-70.

23. Schmidt-Hansen M, Baldwin DR, Hasler E, Zamora J, Abraira V, Roqué Figuls M. PET-CT for assessing mediastinal lymph node involvement in patients with suspected resectable non-small cell lung cancer. Cochrane Database Syst Rev. 2014;13:CD009519. 